\title{
Z-boson production with two unobserved, back-to-back, hard photons at LEP
}

\section{L3 Collaboration}

P. Achard ${ }^{\mathrm{t}}$, O. Adriani ${ }^{\mathrm{y}}$, M. Aguilar-Benitez ${ }^{\mathrm{y}}$, J. Alcaraz ${ }^{\mathrm{y}}$, G. Alemanni ${ }^{\mathrm{w}}$, J. Allaby ${ }^{\mathrm{r}}$, A. Aloisio $^{\text {ac }}$, M.G. Alviggi ac, H. Anderhub ${ }^{\text {aw }}$, V.P. Andreev ${ }^{\text {f,ah }}$, F. Anselmo ${ }^{\text {h }}$, A. Arefiev ${ }^{\text {ab }}$, T. Azemoon ${ }^{\text {c }}$, T. Aziz ${ }^{\text {i }}$, P. Bagnaia ${ }^{\text {am }}$, A. Bajo ${ }^{y}$, G. Baksay ${ }^{z}$, L. Baksay $^{z}$, S.V. Baldew ${ }^{\text {b }}$, S. Banerjee ${ }^{\mathrm{i}}$, Sw. Banerjee ${ }^{\mathrm{d}}$, A. Barczyk ${ }^{\text {aw, au }}$, R. Barillère ${ }^{\mathrm{r}}$, P. Bartalini ${ }^{\mathrm{w}}$, M. Basile ${ }^{\mathrm{h}}$, N. Batalova ${ }^{\text {at }}$, R. Battiston ${ }^{\mathrm{ag}}$, A. Bay ${ }^{\mathrm{w}}$, F. Becattini ${ }^{\mathrm{q}}$, U. Becker ${ }^{\mathrm{m}}$, F. Behner ${ }^{\text {aw }}$, L. Bellucci ${ }^{\mathrm{q}}$, R. Berbeco ${ }^{\mathrm{c}}$, J. Berdugo ${ }^{\mathrm{y}}$, P. Berges ${ }^{\mathrm{m}}$, B. Bertucci ${ }^{\mathrm{ag}}$, B.L. Betev ${ }^{\text {aw }}$, M. Biasini ${ }^{\mathrm{ag}}$, M. Biglietti ${ }^{\text {ac }}$, A. Biland ${ }^{\text {aw }}$, J.J. Blaising ${ }^{\mathrm{d}}$, S.C. Blyth ${ }^{\text {ai }}$, G.J. Bobbink ${ }^{\text {b }}$, A. Böhm ${ }^{\text {a }}$, L. Boldizsar ${ }^{1}$, B. Borgia ${ }^{\text {am }}$, S. Bottai ${ }^{\text {q }}$, D. Bourilkov ${ }^{\text {aw }}$, M. Bourquin ${ }^{\mathrm{t}}$, S. Braccini ${ }^{\mathrm{t}}$, J.G. Branson ${ }^{\text {ao }}$, F. Brochu ${ }^{\mathrm{d}}$, J.D. Burger ${ }^{\mathrm{m}}$, W.J. Burger ${ }^{\mathrm{ag}}$, X.D. Cai ${ }^{\mathrm{m}}$, M. Capell ${ }^{\mathrm{m}}$, G. Cara Romeo ${ }^{\mathrm{h}}$, G. Carlino ${ }^{\text {ac }}$, A. Cartacci ${ }^{\mathrm{q}}$,

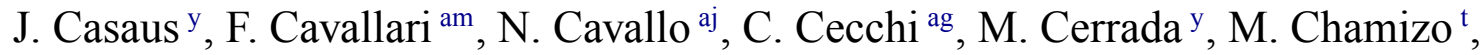
Y.H. Chang ${ }^{\text {ar }}$, M. Chemarin ${ }^{\mathrm{x}}$, A. Chen ${ }^{\text {ar }}$, G. Chen ${ }^{\mathrm{g}}$, G.M. Chen ${ }^{\mathrm{g}}$, H.F. Chen ${ }^{\mathrm{v}}$, H.S. Chen ${ }^{\mathrm{g}}$, G. Chiefari ${ }^{\text {ac }}$, L. Cifarelli ${ }^{\text {an }}$, F. Cindolo ${ }^{\text {h }}$, I. Clare ${ }^{\mathrm{m}}$, R. Clare ${ }^{\text {al }}$, G. Coignet ${ }^{\mathrm{d}}$, N. Colino ${ }^{\mathrm{y}}$, S. Costantini ${ }^{\text {am }}$, B. de la Cruz ${ }^{\mathrm{y}}$, S. Cucciarelli ${ }^{\mathrm{ag}}$, R. de Asmundis ${ }^{\text {ac }}$, P. Déglon ${ }^{\mathrm{t}}, \mathrm{J}$. Debreczeni ${ }^{1}$, A. Degré ${ }^{\mathrm{d}}$, K. Dehmelt ${ }^{\mathrm{z}}$, K. Deiters ${ }^{\mathrm{au}}$, D. della Volpe ${ }^{\text {ac }}$, E. Delmeire ${ }^{\mathrm{t}}$, P. Denes ${ }^{\text {ak }}$, F. DeNotaristefani ${ }^{\text {am }}$, A. De Salvo ${ }^{\text {aw }}$, M. Diemoz ${ }^{\text {am }}$, M. Dierckxsens ${ }^{b}$, C. Dionisi ${ }^{\text {am }}$, M. Dittmar ${ }^{\text {aw }}$, A. Doria ${ }^{\text {ac }}$, M.T. Dova $^{\mathrm{j}, 5}$,

D. Duchesneau ${ }^{d}$, M. Duda ${ }^{a}$, B. Echenard ${ }^{t}$, A. Eline ${ }^{r}$, A. El Hage ${ }^{a}$, H. El Mamouni ${ }^{\mathrm{x}}$, A. Engler ${ }^{\text {ai }}$, F.J. Eppling ${ }^{\mathrm{m}}$, P. Extermann ${ }^{\mathrm{t}}$, M.A. Falagan ${ }^{\mathrm{y}}$, S. Falciano ${ }^{\text {am }}$, A. Favara ${ }^{\text {af }}$, J. Fay ${ }^{\mathrm{x}}$, O. Fedin ${ }^{\text {ah }}$, M. Felcini ${ }^{\text {aw }}$, T. Ferguson ${ }^{\text {ai }}$, H. Fesefeldt ${ }^{\mathrm{a}}$, E. Fiandrini ${ }^{\text {ag }}$, J.H. Field ${ }^{\mathrm{t}}$, F. Filthaut ${ }^{\mathrm{ae}}$, P.H. Fisher ${ }^{\mathrm{m}}$, W. Fisher ${ }^{\text {ak }}$, I. Fisk ${ }^{\mathrm{ao}}$, G. Forconi ${ }^{\mathrm{m}}$, K. Freudenreich ${ }^{\text {aw }}$, C. Furetta ${ }^{\text {aa }}$, Yu. Galaktionov ${ }^{\text {ab,m }}$, S.N. Ganguli ${ }^{i}$, P. Garcia-Abia ${ }^{y}$, M. Gataullin ${ }^{\text {af }}$, S. Gentile ${ }^{\text {am }}$, S. Giagu ${ }^{\text {am }}$, Z.F. Gong ${ }^{\mathrm{v}}$, G. Grenier ${ }^{\mathrm{x}}$, O. Grimm ${ }^{\mathrm{aw}}$, M.W. Gruenewald ${ }^{\mathrm{p}}$, M. Guida ${ }^{\text {an }}$, V.K. Gupta ${ }^{\mathrm{ak}}$, A. Gurtu ${ }^{\mathrm{i}}$, L.J. Gutay ${ }^{\text {at }}$, D. Haas ${ }^{\mathrm{e}}$, D. Hatzifotiadou ${ }^{\text {h }}$, T. Hebbeker ${ }^{\text {a }}$, A. Hervé ${ }^{r}$, J. Hirschfelder ${ }^{\text {ai }}$, H. Hofer ${ }^{\text {aw }}$, M. Hohlmann ${ }^{\mathrm{z}}$, G. Holzner ${ }^{\text {aw }}$, S.R. Hou ${ }^{\text {ar }}$, B.N. Jin ${ }^{\mathrm{g}}$, P. Jindal ${ }^{\mathrm{n}}$, L.W. Jones ${ }^{\mathrm{c}}$, 
P. de Jong ${ }^{\text {b }}$, I. Josa-Mutuberría ${ }^{y}$, M. Kaur ${ }^{\text {n }}$, M.N. Kienzle-Focacci ${ }^{\text {t }}$ J.K. Kim ${ }^{\text {aq }}$, J. Kirkby ${ }^{\mathrm{r}}$, W. Kittel ${ }^{\text {ae }}$, A. Klimentov ${ }^{\mathrm{m}, \mathrm{ab}}$, A.C. König ${ }^{\text {ae }}$, M. Kopal ${ }^{\text {at }}$, V. Koutsenko ${ }^{\mathrm{m}, \mathrm{ab}}$, M. Kräber ${ }^{\text {aw }}$, R.W. Kraemer ${ }^{\text {ai }}$, A. Krüger ${ }^{\text {av }}$, A. Kunin ${ }^{\text {m }}$, P. Ladron de Guevara ${ }^{y}$, I. Laktineh ${ }^{\mathrm{x}}$, G. Landi ${ }^{\mathrm{q}}$, M. Lebeau ${ }^{\mathrm{r}}$, A. Lebedev ${ }^{\mathrm{m}}$, P. Lebrun ${ }^{\mathrm{x}}$, P. Lecomte ${ }^{\text {aw }}$, P. Lecoq ${ }^{r}$, P. Le Coultre ${ }^{\text {aw }}$, J.M. Le Goff ${ }^{\mathrm{r}}$, R. Leiste ${ }^{\text {av }}$, M. Levtchenko ${ }^{\text {aa }}$, P. Levtchenko ah ${ }^{\text {ah }}$ C. Li ${ }^{v}$, S. Likhoded ${ }^{\text {av }}$, C.H. Lin ${ }^{\text {ar }}$, W.T. Lin ${ }^{\text {ar }}$, F.L. Linde ${ }^{\text {b }}$, L. Lista ${ }^{\text {ac }}$, Z.A. Liu ${ }^{\mathrm{g}}$, W. Lohmann ${ }^{\text {av }}$, E. Longo ${ }^{\text {am }}$, Y.S. Lu ${ }^{\mathrm{g}}$, C. Luci ${ }^{\text {am }}$, L. Luminari ${ }^{\text {am }}$, W. Lustermann ${ }^{\text {aw }}$, W.G. Ma ${ }^{\mathrm{v}}$, L. Malgeri ${ }^{\mathrm{r}}$, A. Malinin ${ }^{\mathrm{ab}}$, C. Maña ${ }^{\mathrm{y}}$, J. Mans $^{\text {ak }}$, J.P. Martin ${ }^{\mathrm{x}}$, F. Marzano ${ }^{\mathrm{am}}$, A. Matyja ${ }^{\mathrm{r}}$, K. Mazumdar ${ }^{\mathrm{i}}$, R.R. McNeil ${ }^{\mathrm{f}}$, S. Mele ${ }^{\mathrm{r}, \mathrm{ac}}$, L. Merola $^{\text {ac }}$, M. Meschini ${ }^{\text {q, W.J. Metzger }}{ }^{\text {ae }}$, A. Mihul ${ }^{\text {k }}$, H. Milcent ${ }^{\mathrm{r}}$, G. Mirabelli ${ }^{\text {am }}$, J. Mnich $^{\mathrm{a}}$, G.B. Mohanty ${ }^{\mathrm{i}}$, G.S. Muanza ${ }^{\mathrm{x}}$, A.J.M. Muijs ${ }^{\mathrm{b}}$, B. Musicar ${ }^{\mathrm{ao}}$, M. Musy ${ }^{\text {am }}$, S. Nagy ${ }^{\circ}$, S. Natale $^{t}$, M. Napolitano ${ }^{\text {ac }}$, F. Nessi-Tedaldi ${ }^{\text {aw }}$, H. Newman ${ }^{\text {af }}$, A. Nisati ${ }^{\text {am }}$, T. Novak ${ }^{\text {ae }}$, H. Nowak ${ }^{\text {av }}$, R. Ofierzynski ${ }^{\text {aw }}$, G. Organtini ${ }^{\text {am }}$, I. Pal ${ }^{\text {at }}$, C. Palomares ${ }^{y}$, P. Paolucci ${ }^{\text {ac }}$, R. Paramatti ${ }^{\text {am }}$, G. Passaleva ${ }^{\mathrm{q}}$, S. Patricelli ${ }^{\text {ac }}$, T. Paul $^{\mathrm{j}}$, M. Pauluzzi $^{\mathrm{ag}}$, C. Paus ${ }^{\mathrm{m}}$, F. Pauss ${ }^{\text {aw }}$, M. Pedace ${ }^{\mathrm{am}}$, S. Pensotti ${ }^{\text {aa }}$, D. Perret-Gallix ${ }^{\mathrm{d}}$, D. Piccolo ${ }^{\text {ac }}$, F. Pierella $^{\text {h }}$, M. Pioppi ${ }^{\text {ag }}$, P.A. Piroué ${ }^{\text {ak }}$, E. Pistolesi $^{\text {aa }}$, V. Plyaskin ${ }^{\text {ab }}$, M. Pohl ${ }^{\text {t }}$, V. Pojidaev ${ }^{\mathrm{q}}$, J. Pothier ${ }^{\mathrm{r}}$, D. Prokofiev ${ }^{\text {ah }}$, G. Rahal-Callot ${ }^{\text {aw }}$, M.A. Rahaman ${ }^{\mathrm{i}}$, P. Raics ${ }^{\text {o }}$, N. Raja ${ }^{\mathrm{i}}$, R. Ramelli ${ }^{\text {aw }}$, P.G. Rancoita ${ }^{\text {aa }}$, R. Ranieri ${ }^{\mathrm{q}}$, A. Raspereza ${ }^{\text {av }}$, P. Razis ${ }^{\text {ad }}$, D. Ren ${ }^{\text {aw }}$, M. Rescigno ${ }^{\text {am }}$, S. Reucroft ${ }^{\mathrm{j}}$, S. Riemann ${ }^{\text {av }}$, K. Riles ${ }^{\text {c }}$, B.P. Roe ${ }^{\mathrm{c}}$, L. Romero ${ }^{\mathrm{y}}$, A. Rosca ${ }^{\text {av }}$, C. Rosemann ${ }^{\mathrm{a}}$, C. Rosenbleck ${ }^{\mathrm{a}}$, S. Rosier-Lees ${ }^{\mathrm{d}}$, S. Roth ${ }^{\mathrm{a}}$, J.A. Rubio ${ }^{\text {r }}$, G. Ruggiero ${ }^{\text {q }}$, H. Rykaczewski ${ }^{\text {aw }}$, A. Sakharov ${ }^{\text {aw }}$, S. Saremi ${ }^{\text {f }}$, S. Sarkar ${ }^{\text {am }}$, J. Salicio $^{\text {r }}$, E. Sanchez ${ }^{y}$, C. Schäfer ${ }^{r}$, V. Schegelsky ${ }^{\text {ah }}$, H. Schopper ${ }^{\text {u }}$, D.J. Schotanus ${ }^{\text {ae }}$, C. Sciacca ${ }^{\text {ac }}$, L. Servoli ${ }^{\text {ag }}$, S. Shevchenko ${ }^{\text {af }}$, N. Shivarov ap, V. Shoutko ${ }^{\mathrm{m}}$, E. Shumilov ${ }^{\mathrm{ab}}$, A. Shvorob ${ }^{\text {af }}$, D. Son ${ }^{\mathrm{aq}}$, C. Souga ${ }^{\mathrm{x}}$, P. Spillantini ${ }^{\mathrm{q}}$, M. Steuer ${ }^{\mathrm{m}}$, D.P. Stickland ${ }^{\text {ak }}$, B. Stoyanov ap, A. Straessner ${ }^{\mathrm{t}}$, K. Sudhakar ${ }^{\mathrm{i}}$, G. Sultanov ${ }^{\text {ap }}$, L.Z. Sun ${ }^{\mathrm{v}}$, S. Sushkov ${ }^{\mathrm{a}}$, H. Suter ${ }^{\text {aw }}$, J.D. Swain ${ }^{\mathrm{j}}$, Z. Szillasi ${ }^{\mathrm{z}, 3}$, X.W. Tang ${ }^{\mathrm{g}}$, P. Tarjan ${ }^{\mathrm{o}}$, L. Tauscher ${ }^{\mathrm{e}}$, L. Taylor ${ }^{\mathrm{j}}$, B. Tellili ${ }^{\mathrm{x}}, \mathrm{D}$. Teyssier ${ }^{\mathrm{x}}$, C. Timmermans ${ }^{\text {ae }}$, Samuel C.C. Ting ${ }^{\mathrm{m}}$, S.M. Ting ${ }^{\mathrm{m}}$, S.C. Tonwar ${ }^{\mathrm{i}}$, J. Tóth ${ }^{1}$, C. Tully ${ }^{\mathrm{ak}}$, K.L. Tung ${ }^{\mathrm{g}}$, J. Ulbricht ${ }^{\text {aw }}$, E. Valente ${ }^{\text {am }}$, R.T. Van de Walle ${ }^{\text {ae }}$, R. Vasquez ${ }^{\text {at }}$, V. Veszpremi ${ }^{\text {z }}$, G. Vesztergombi ${ }^{1}$, I. Vetlitsky ${ }^{\text {ab }}$, G. Viertel ${ }^{\text {aw }}$, S. Villa ${ }^{\text {al }}$, M. Vivargent ${ }^{\text {d }}$, S. Vlachos ${ }^{\mathrm{e}}$, I. Vodopianov $^{z}$, H. Vogel ${ }^{\text {ai }}, H$. Vogt ${ }^{\text {av }}$, I. Vorobiev ${ }^{\text {ai,ab }}$, A.A. Vorobyov ${ }^{\text {ah }}$, M. Wadhwa ${ }^{\mathrm{e}}$, Q. Wang ${ }^{\text {ae }}$, X.L. Wang ${ }^{\mathrm{v}}$, Z.M. Wang ${ }^{\mathrm{v}}$, M. Weber ${ }^{\mathrm{r}}$, S. Wynhoff ${ }^{\mathrm{ak}}$, L. Xia ${ }^{\text {af }}$, Z.Z. Xu ${ }^{\mathrm{v}}$,

J. Yamamoto ${ }^{\mathrm{c}}$, B.Z. Yang ${ }^{\mathrm{v}}$, C.G. Yang ${ }^{\mathrm{g}}$, H.J. Yang ${ }^{\mathrm{c}}$, M. Yang ${ }^{\mathrm{g}}$, S.C. Yeh ${ }^{\text {as }}$, An. Zalite ${ }^{\text {ah }}$, Yu. Zalite ${ }^{\text {ah }}$, Z.P. Zhang ${ }^{v}$, J. Zhao ${ }^{v}$, G.Y. Zhu ${ }^{\mathrm{g}}$, R.Y. Zhu ${ }^{\text {af }}$, H.L. Zhuang ${ }^{\mathrm{g}}$, A. Zichichi ${ }^{\mathrm{h}, \mathrm{r}, \mathrm{s}}$, B. Zimmermann ${ }^{\text {aw }}$, M. Zöller ${ }^{\mathrm{a}}$

\footnotetext{
a III. Physikalisches Institut, RWTH, D-52056 Aachen, Germany ${ }^{1}$

${ }^{\mathrm{b}}$ National Institute for High Energy Physics, NIKHEF, and University of Amsterdam, NL-1009 DB Amsterdam, The Netherlands c University of Michigan, Ann Arbor, MI 48109, USA

d Laboratoire d'Annecy-le-Vieux de Physique des Particules, LAPP, IN2P3-CNRS, BP 110, F-74941 Annecy-le-Vieux cedex, France
} 
e Institute of Physics, University of Basel, CH-4056 Basel, Switzerland

${ }^{\mathrm{f}}$ Louisiana State University, Baton Rouge, LA 70803, USA

g Institute of High Energy Physics, IHEP, 100039 Beijing, China 6

${ }^{\mathrm{h}}$ University of Bologna and INFN, Sezione di Bologna, I-40126 Bologna, Italy

${ }^{\mathrm{i}}$ Tata Institute of Fundamental Research, Mumbai (Bombay) 400 005, India

$\mathrm{j}$ Northeastern University, Boston, MA 02115, USA

${ }^{\mathrm{k}}$ Institute of Atomic Physics and University of Bucharest, R-76900 Bucharest, Romania

${ }^{1}$ Central Research Institute for Physics of the Hungarian Academy of Sciences, H-1525 Budapest 114, Hungary ${ }^{2}$

$\mathrm{m}$ Massachusetts Institute of Technology, Cambridge, MA 02139, USA

${ }^{\mathrm{n}}$ Panjab University, Chandigarh 160 014, India

${ }^{\circ}$ KLTE-ATOMKI, H-4010 Debrecen, Hungary ${ }^{3}$

$\mathrm{p}$ Department of Experimental Physics, University College Dublin, Belfield, Dublin 4, Ireland

q INFN, Sezione di Firenze and University of Florence, I-50125 Florence, Italy

${ }^{\mathrm{r}}$ European Laboratory for Particle Physics, CERN, CH-1211 Geneva 23, Switzerland

s World Laboratory, FBLJA Project, CH-1211 Geneva 23, Switzerland

${ }^{\mathrm{t}}$ University of Geneva, CH-1211 Geneva 4, Switzerland

u University of Hamburg, D-22761 Hamburg, Germany

${ }^{v}$ Chinese University of Science and Technology, USTC, Hefei, Anhui 230 029, China ${ }^{6}$

${ }^{w}$ University of Lausanne, CH-1015 Lausanne, Switzerland

x Institut de Physique Nucléaire de Lyon, IN2P3-CNRS, Université Claude Bernard, F-69622 Villeurbanne, France

y Centro de Investigaciones Energéticas, Medioambientales y Tecnológicas, CIEMAT, E-28040 Madrid, Spain ${ }^{4}$

${ }^{\mathrm{z}}$ Florida Institute of Technology, Melbourne, FL 32901, USA

aa INFN, Sezione di Milano, I-20133 Milan, Italy

ab Institute of Theoretical and Experimental Physics, ITEP, Moscow, Russia

${ }^{\text {ac }}$ INFN, Sezione di Napoli and University of Naples, I-80125 Naples, Italy

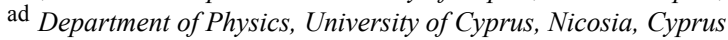

ae Radboud University and NIKHEF, NL-6525 ED Nijmegen, The Netherlands

af California Institute of Technology, Pasadena, CA 91125, USA

ag INFN, Sezione di Perugia and Università Degli Studi di Perugia, I-06100 Perugia, Italy

ah Nuclear Physics Institute, St. Petersburg, Russia

ai Carnegie Mellon University, Pittsburgh, PA 15213, USA

aj INFN, Sezione di Napoli and University of Potenza, I-85100 Potenza, Italy

${ }^{\mathrm{ak}}$ Princeton University, Princeton, NJ 08544, USA

al University of Californa, Riverside, CA 92521, USA

am INFN, Sezione di Roma and University of Rome, "La Sapienza", I-00185 Rome, Italy

an University and INFN, Salerno, I-84100 Salerno, Italy

ao University of California, San Diego, CA 92093, USA

ap Bulgarian Academy of Sciences, Central Lab. of Mechatronics and Instrumentation, BU-1113 Sofia, Bulgaria

aq The Center for High Energy Physics, Kyungpook National University, 702-701 Taegu, Republic of Korea

ar National Central University, Chung-Li, Taiwan

as Department of Physics, National Tsing Hua University, Taiwan

at Purdue University, West Lafayette, IN 47907, USA

au Paul Scherrer Institut, PSI, CH-5232 Villigen, Switzerland av DESY, D-15738 Zeuthen, Germany

aw Eidgenössische Technische Hochschule, ETH Zürich, CH-8093 Zürich, Switzerland

Received 1 March 2005; received in revised form 11 March 2005; accepted 15 March 2005

Available online 21 March 2005

Editor: L. Rolandi

\section{Abstract}

The double-radiative process $\mathrm{e}^{+} \mathrm{e}^{-} \rightarrow \mathrm{Z} \gamma \gamma \rightarrow \mathrm{q} \overline{\mathrm{q}} \gamma \gamma$ where the two hard photons escape detection at low polar angles into opposite directions, is studied in $0.62 \mathrm{fb}^{-1}$ of data collected with the L3 detector at LEP at centre-of-mass energies 
between 188.6 and $209.2 \mathrm{GeV}$. The cross sections are measured and found to be consistent with the Standard Model expectations.

(C) 2005 Published by Elsevier B.V.

\section{Introduction}

One of the most copious sources of events in $\mathrm{e}^{+} \mathrm{e}^{-}$ collisions at LEP above the $\mathrm{Z}$ resonance is the process $\mathrm{e}^{+} \mathrm{e}^{-} \rightarrow \mathrm{q} \overline{\mathrm{q}}$, with a cross section of about $100 \mathrm{pb}$. The effective centre-of-mass energy, $\sqrt{s^{\prime}}$, at which this hadron production takes place does not necessarily correspond to the centre-of-mass energy of the LEP machine, $\sqrt{s}$, owing to the emission of one or more hard initial-state-radiation (ISR) photons by the incoming electrons or positrons. These photons are most likely emitted along the beam line, in the low polar-angle regions of the detectors which are not instrumented and, therefore, escape detection.

The cross section of the $\mathrm{e}^{+} \mathrm{e}^{-} \rightarrow \mathrm{q} \overline{\mathrm{q}}$ process was measured $[1,2]$ and found to be in agreement with the Standard Model predictions for both a subsample of events with values of $\sqrt{s^{\prime}}$ close to $\sqrt{s}$ and a more inclusive sample extending to lower values of $\sqrt{s^{\prime}}$. The emission of ISR photons often implies $\sqrt{s^{\prime}} \approx m_{Z}$, where $m_{Z}=91.19 \mathrm{GeV}$ is the mass of the $\mathrm{Z}$ boson. This phenomenon is commonly called "radiative return to the $\mathrm{Z}$ ". The process $\mathrm{e}^{+} \mathrm{e}^{-} \rightarrow \mathrm{Z} \gamma \rightarrow \mathrm{q} \overline{\mathrm{q}} \gamma$, where a hard ISR photon is responsible for such radiative return to the Z, was studied in detail [4,5]. Events in which the photon was visible in the detector, were used to constrain possible anomalous triple-couplings between neutral gauge bosons [4]. Events with either a detected photon or a low-angle undetected photon

\footnotetext{
${ }^{1}$ Supported by the German Bundesministerium für Bildung, Wissenschaft, Forschung und Technologie.

2 Supported by the Hungarian OTKA fund under contract Nos. T019181, F023259 and T037350.

3 Also supported by the Hungarian OTKA fund under contract No. T026178.

${ }^{4}$ Supported also by the Comisión Interministerial de Ciencia y Tecnología.

5 Also supported by CONICET and Universidad Nacional de La Plata, CC 67, 1900 La Plata, Argentina.

6 Supported by the National Natural Science Foundation of China.
}

were used to reconstruct the mass of the $\mathrm{Z}$ boson and validate the analysis tools used in the measurement of the mass of the $\mathrm{W}$ boson [5]. The $\mathrm{e}^{+} \mathrm{e}^{-} \rightarrow \mathrm{Z} \gamma \gamma \rightarrow$ $\mathrm{q} \overline{\mathrm{q}} \gamma \gamma$ process, where both ISR photons were visible in the detector, was first observed by the L3 Collaboration [6]. The cross section of this process was then measured for $\sqrt{s}=130-209 \mathrm{GeV}$ and found to be in agreement with the predictions [7].

This Letter extends the study of the $\mathrm{e}^{+} \mathrm{e}^{-} \rightarrow$ $\mathrm{Z} \gamma \gamma \rightarrow \mathrm{q} \overline{\mathrm{q}} \gamma \gamma$ process to the case in which both ISR photons are emitted at low polar angles and are therefore not detected. In particular, the case is considered in which the two photons are emitted on opposite sides of the detector, with comparable transverse momenta. In this topology, the two jets originating from the Z-boson decay are back-to-back. In the following, this process is denoted as "double-radiative return to the Z". This study complements the previous studies of the $\mathrm{e}^{+} \mathrm{e}^{-} \rightarrow \mathrm{q} \overline{\mathrm{q}}$ process in a very specific phase-space region and allows further tests of Monte Carlo simulations of ISR photons in hadronic events. Moreover, final states with two back-to-back hadronic jets and missing energy are a signature of the near-threshold production of the Standard Model Higgs boson, $\mathrm{H}$, at LEP in the reaction $\mathrm{e}^{+} \mathrm{e}^{-} \rightarrow \mathrm{ZH}$. In this case, the missing energy is due to a $\mathrm{Z}$ boson decaying into neutrinos and the jets to the Higgs boson. In addition, manifestations of new physics in the production of an invisibly-decaying Higgs boson in association with a $\mathrm{Z}$ boson decaying into hadrons would also give rise to the same final state. Finally, similar event topologies are predicted by supersymmetry. Therefore, a study of double-radiative return to the $\mathrm{Z}$ with unobserved photons validates the background Monte Carlo simulations for those searches.

This analysis selects $\mathrm{e}^{+} \mathrm{e}^{-} \rightarrow \mathrm{q} \overline{\mathrm{q}}$ events with two or more hard ISR photons satisfying the following phasespace criteria:

$$
\begin{aligned}
& E_{\gamma_{1,2}}>5 \mathrm{GeV}, \\
& \left|\cos \theta_{\gamma_{1,2}}\right| \geqslant 0.96,
\end{aligned}
$$



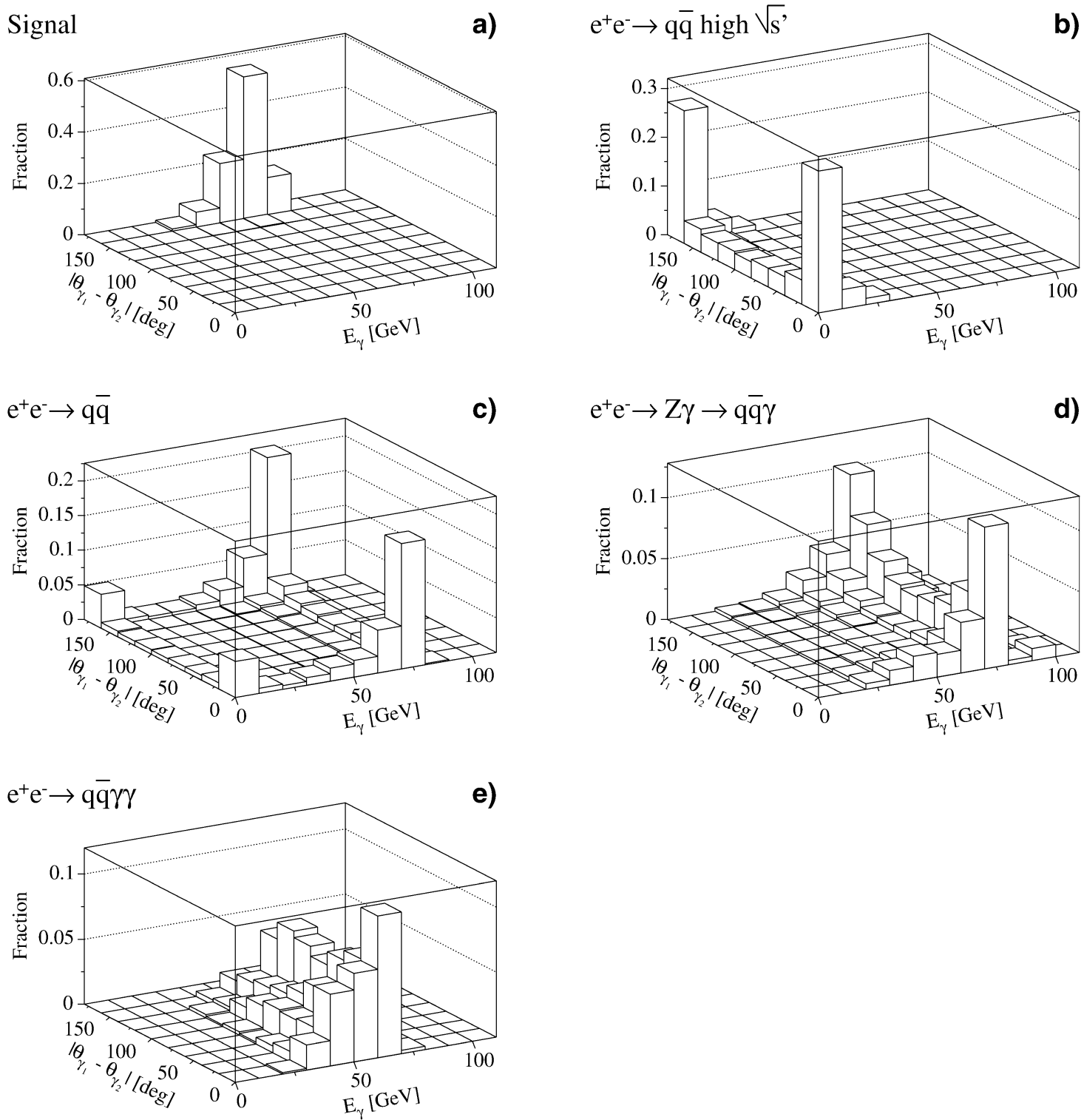

Fig. 1. Distributions at generator level of the absolute difference of the two photon polar angles versus the energy of the most energetic photon for (a) the signal, (b) the $\mathrm{e}^{+} \mathrm{e}^{-} \rightarrow \mathrm{qq}$ process for $\sqrt{s^{\prime}} / \sqrt{s}>0.85$ and $\sqrt{s^{\prime}}>60 \mathrm{GeV}$, (c) the $\mathrm{e}^{+} \mathrm{e}^{-} \rightarrow \mathrm{qq}$ process for $\sqrt{s^{\prime}}>60 \mathrm{GeV},(\mathrm{d})$ the $\mathrm{e}^{+} \mathrm{e}^{-} \rightarrow \mathrm{Z} \gamma \rightarrow \mathrm{q} \overline{\mathrm{q}} \gamma$ process, and (e) the $\mathrm{e}^{+} \mathrm{e}^{-} \rightarrow \mathrm{Z} \gamma \gamma \rightarrow \mathrm{q} \overline{\mathrm{q}} \gamma \gamma$ process. Only events with at least two photons with energies greater than $1 \mathrm{MeV}$ are shown, for a sample at $\sqrt{s}=189 \mathrm{GeV}$. The histograms show the fraction of the cross section of each process in each bin. These cross sections are, respectively, 5.8, 89.7, 16.1, 20.3 and $0.4 \mathrm{pb}$.

$\left|\sqrt{s^{\prime}}-m_{\mathrm{Z}}\right|<2 \Gamma_{\mathrm{Z}}$

$\cos \theta_{\gamma_{1}} \cos \theta_{\gamma_{2}}<0$

$\left|p_{\gamma_{1}}^{\mathrm{T}}-p_{\gamma_{2}}^{\mathrm{T}}\right|<0.1 \sqrt{s}$ where $E_{\gamma_{i}}, \theta_{\gamma_{i}}$ and $p_{\gamma_{i}}^{\mathrm{T}}$ are the energy, polar angle and momentum in the plane transverse to the beams of the photon $i$, respectively. $\Gamma_{\mathrm{Z}}$ denotes the width of the $\mathrm{Z}$ boson, $2.49 \mathrm{GeV}$ [3]. If more than two ISR photons are present in the event, this signal definition is applied to the two most energetic ones. These criteria 
select about $6 \%$ of the phase space of the $\mathrm{e}^{+} \mathrm{e}^{-} \rightarrow \mathrm{q} \overline{\mathrm{q}}$ process, corresponding to a cross section of about 5.5 $\mathrm{pb}$ in the $\sqrt{s}$ range explored at LEP. Fig. 1 illustrates the complementarity of this phase space with those covered by the analyses of the $\mathrm{e}^{+} \mathrm{e}^{-} \rightarrow \mathrm{q} \overline{\mathrm{q}}, \mathrm{e}^{+} \mathrm{e}^{-} \rightarrow$ $\mathrm{Z} \gamma \rightarrow \mathrm{q} \overline{\mathrm{q}} \gamma$ and $\mathrm{e}^{+} \mathrm{e}^{-} \rightarrow \mathrm{Z} \gamma \gamma \rightarrow \mathrm{q} \overline{\mathrm{q}} \gamma \gamma$ processes described in Refs. [1,4,7].

\section{Data and Monte Carlo samples}

This measurement is based on $0.62 \mathrm{fb}^{-1}$ of data collected with the L3 detector [8] at LEP in the years from 1998 through 2000 at centre-of-mass energies between $\sqrt{s}=188.6 \mathrm{GeV}$ and $\sqrt{s}=209.2 \mathrm{GeV}$, as detailed in Table 1. In the last year of data taking, the LEP centre-of-mass energy was routinely increased while the beams were colliding in order to enhance the sensitivity of the search for the Standard Model Higgs boson, exploring the range $\sqrt{s}=202.5-209.2 \mathrm{GeV}$. In the following, this last data sample is split into two energy ranges.

The KK2f [10] Monte Carlo program is used, with default options, to generate a total of 1.9 million $\mathrm{e}^{+} \mathrm{e}^{-} \rightarrow \mathrm{q} \overline{\mathrm{q}}$ events which can contain one or more hard ISR photons, at the centre-of-mass energies listed in Table 1. These events correspond to about 35 times the luminosity of the data and cover a phase space much larger than that of the criteria (1)-(5). If at least two ISR photons which satisfy the criteria (1)-(5) are present in an event this is treated as signal, otherwise it is considered as background. This distinction between signal and background is performed on generated variables, before any event simulation and any application of detector resolutions.

Other background processes are generated with the Monte Carlo programs PYTHIA [9] for $\mathrm{e}^{+} \mathrm{e}^{-} \rightarrow$ $\mathrm{Ze}^{+} \mathrm{e}^{-}$and $\mathrm{e}^{+} \mathrm{e}^{-} \rightarrow$ ZZ, KK2f for $\mathrm{e}^{+} \mathrm{e}^{-} \rightarrow \tau^{+} \tau^{-}$, PHOJET [11] for hadron production in two-photon collisions and KORALW [12] for W-boson pair production except for $\mathrm{e}_{\mathrm{e}} \mathrm{q} \overline{\mathrm{q}}^{\prime}$ final states, generated with
EXCALIBUR [13]. The hadronisation process for signal and background events is modelled with the JETSET [9] program.

The L3 detector response is simulated using the GEANT [14] and GHEISHA [15] programs, which model the effects of energy loss, multiple scattering and showering in the detector. Time-dependent detector efficiencies, as monitored during data-taking periods, are also simulated.

\section{Event selection}

The event selection proceeds from a sample of high-multiplicity events. Events containing photons, electrons or muons with energies above $20 \mathrm{GeV}$ are removed in order to reduce the backgrounds from $\mathrm{e}^{+} \mathrm{e}^{-} \rightarrow \mathrm{q} \overline{\mathrm{q}}$ events with ISR photons in the detector and events containing $\mathrm{W}$ bosons which decay into leptons. The visible mass, $M_{\mathrm{vis}}$, and the visible energy, $E_{\mathrm{vis}}$, of these events are required to satisfy $50<$ $M_{\text {vis }}<140 \mathrm{GeV}$ and $0.4<E_{\mathrm{vis}} / \sqrt{s}<0.65$, to reduce both $\mathrm{e}^{+} \mathrm{e}^{-} \rightarrow \mathrm{q} \overline{\mathrm{q}}$ events without missing energy due to ISR photons and most events from two-photon collisions. The latter cut is illustrated in Fig. 2(a). Events from two-photon collisions are further suppressed by requiring $\left|\cos \theta_{\text {thrust }}\right|<0.96$, where $\theta_{\text {thrust }}$ is the angle between the thrust axis and the beam line. Events are then reconstructed into two jets by means of the DURHAM algorithm [16] and the signal signature of two back-to-back jets is enforced by requiring the angle between the two jets, $\theta_{\mathrm{jj}}$, to satisfy $\theta_{\mathrm{jj}}>1.5 \mathrm{rad}$. Finally, the sum of the momenta of the two jets in the plane transverse to the beams, $p_{\mathrm{T}}$, must be less than $0.2 E_{\text {vis }}$. This cut, shown in Fig. 2(b), accounts for the fact that all missing momentum in signal events is due to the two ISR photons nearly collinear with the beam particles and therefore directed along the beam line. After this preselection, 17208 events are selected in data, well consistent with the 17151 events expected from Monte Carlo simulations, of which 13\% are from

Table 1

Centre-of-mass energies and corresponding integrated luminosities, $\mathcal{L}$, considered in this analysis. The last two energy ranges correspond to the average centre-of-mass energy values $\langle\sqrt{s}\rangle=204.8 \mathrm{GeV}$ and $\langle\sqrt{s}\rangle=206.6 \mathrm{GeV}$, respectively

\begin{tabular}{llllllll}
\hline$\sqrt{s}(\mathrm{GeV})$ & 188.6 & 191.6 & 195.6 & 199.5 & 201.7 & $202.5-205.5$ & $205.5-209.2$ \\
\hline $\mathcal{L}\left(\mathrm{pb}^{-1}\right)$ & 176.0 & 29.5 & 83.4 & 81.4 & 36.7 & 77.5 & 138.6 \\
\hline
\end{tabular}



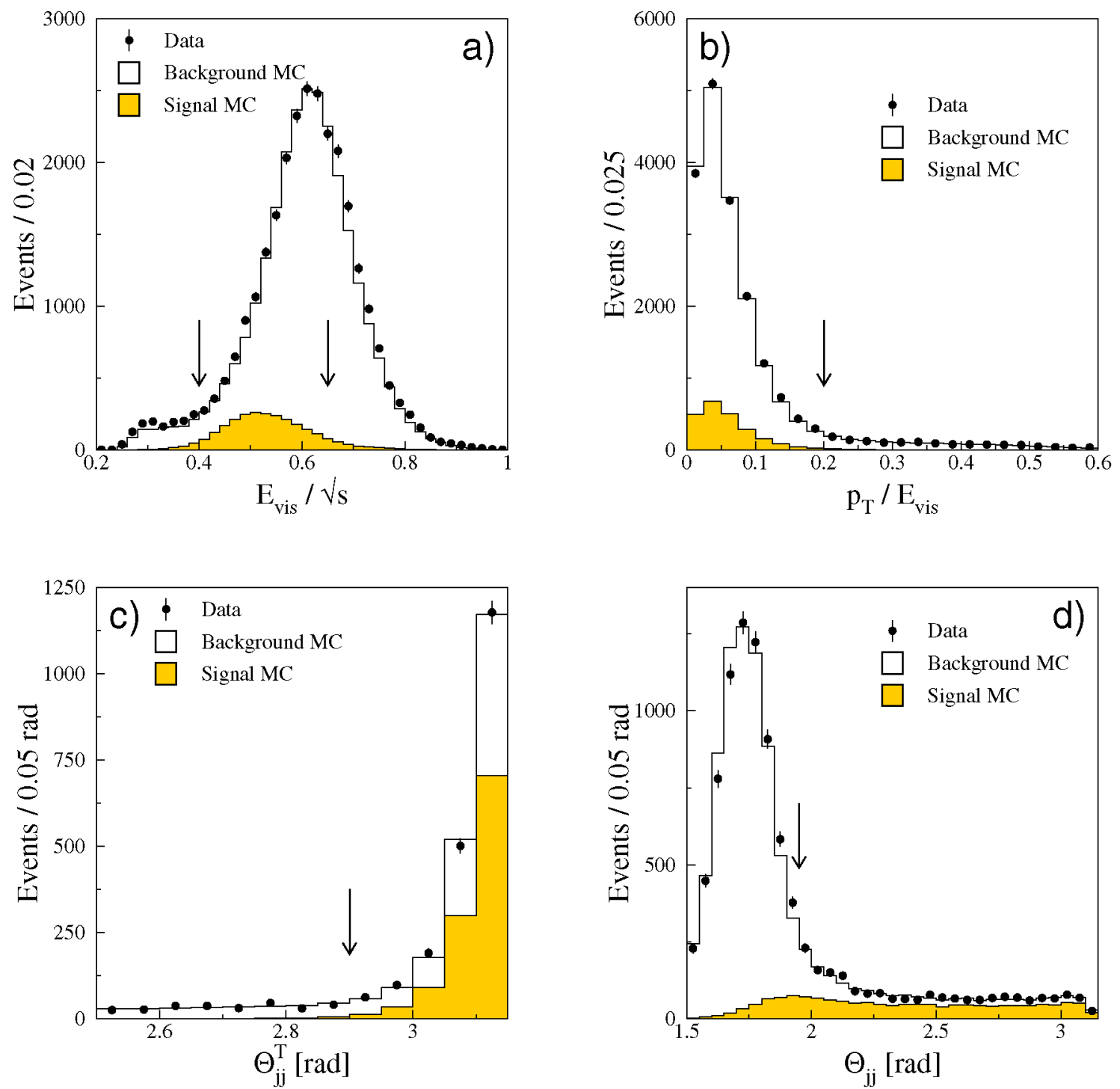

Fig. 2. Distribution for data and Monte Carlo of (a) the visible energy divided by the centre-of-mass energy; (b) the sum of the transverse momenta of the two jets divided by the visible energy; (c) the angle between the two jets in the plane transverse to the beams; (d) the angle between the jets. The distributions in (a) and (b) refer to preselection level, those in (c) and (d) to the final selection. The arrows represent the position of the cuts, once all other preselection or selection cuts are applied.

signal and $87 \%$ from background. The background is almost entirely composed by $\mathrm{e}^{+} \mathrm{e}^{-} \rightarrow \mathrm{q} \overline{\mathrm{q}}$ events which do not satisfy the signal definition (1)-(5). Small contributions arise from four-fermion production and hadron production in two-photon collisions. The signal efficiency at this stage of the analysis is $68 \%$.

Three additional cuts are devised to reduce the residual background and enhance the signal compo- nent in this sample. The energy of the most energetic jet must be greater than $0.4 \sqrt{s}$; the angle between the two jets in the plane transverse to the beams, $\theta_{\mathrm{jj}}^{\mathrm{T}}$, is required to be $\theta_{\mathrm{jj}}^{\mathrm{T}}>2.9 \mathrm{rad}$, as shown in Fig. 2(c); the polar angle of the jet closest to the beam line, $\theta_{\text {low }}^{\text {jet }}$, should be such that $\left|\cos \theta_{\text {low }}^{\text {jet }}\right|<0.85$. Finally, two of the preselection criteria are tightened: $\theta_{\mathrm{jj}}>1.95 \mathrm{rad}$ 
Table 2

Number of events observed in data after the final selection, $N_{\text {Data }}$, compared with the total number of events expected from Monte Carlo, $N_{\mathrm{MC}}$. The number of signal events expected from the KK2f Monte Carlo, $N_{\text {Sign }}$, is also given, together with the number of background events, $N_{\text {Back }}$. The selection efficiency, $\varepsilon$, is also listed, together with the measured, $\sigma$, and expected, $\sigma_{\mathrm{th}}$, signal cross sections. The uncertainties on $N_{\mathrm{MC}}$, $N_{\text {Sign }}, N_{\text {Back }}$ and $\varepsilon$ correspond to the statistical uncertainty of the Monte Carlo. The first uncertainty on $\sigma$ is statistical, the second systematic

\begin{tabular}{|c|c|c|c|c|c|c|c|}
\hline $\begin{array}{l}\sqrt{s} \\
(\mathrm{GeV})\end{array}$ & $N_{\text {Data }}$ & $N_{\mathrm{MC}}$ & $N_{\text {Sign }}$ & $N_{\text {Back }}$ & $\begin{array}{l}\varepsilon \\
(\%)\end{array}$ & $\begin{array}{l}\sigma \\
(\mathrm{pb})\end{array}$ & $\begin{array}{l}\sigma_{\text {th }} \\
(\mathrm{pb})\end{array}$ \\
\hline 188.6 & 632 & $660.1 \pm 6.9$ & $372.3 \pm 4.6$ & $287.9 \pm 5.1$ & $36.1 \pm 0.4$ & $5.31 \pm 0.39 \pm 0.37$ & 5.9 \\
\hline 191.6 & 84 & $97.3 \pm 2.1$ & $56.7 \pm 1.5$ & $40.6 \pm 1.5$ & $34.7 \pm 0.9$ & $3.63 \pm 0.88 \pm 0.44$ & 5.7 \\
\hline 195.5 & 238 & $236.5 \pm 4.2$ & $149.7 \pm 2.8$ & $86.8 \pm 3.1$ & $32.3 \pm 0.6$ & $5.51 \pm 0.55 \pm 0.32$ & 5.5 \\
\hline 199.5 & 216 & $196.3 \pm 3.9$ & $126.6 \pm 2.5$ & $69.6 \pm 3.0$ & $29.6 \pm 0.6$ & $6.05 \pm 0.60 \pm 0.31$ & 5.3 \\
\hline 201.7 & 82 & $80.9 \pm 2.1$ & $52.8 \pm 1.6$ & $28.1 \pm 1.4$ & $27.8 \pm 0.8$ & $5.32 \pm 0.87 \pm 0.33$ & 5.2 \\
\hline 204.8 & 147 & $153.2 \pm 1.6$ & $102.2 \pm 1.0$ & $50.9 \pm 1.2$ & $26.4 \pm 0.3$ & $4.81 \pm 0.58 \pm 0.27$ & 5.1 \\
\hline 206.6 & 273 & $259.9 \pm 2.3$ & $173.5 \pm 1.3$ & $86.5 \pm 2.0$ & $25.5 \pm 0.2$ & $5.33 \pm 0.46 \pm 0.27$ & 5.0 \\
\hline
\end{tabular}

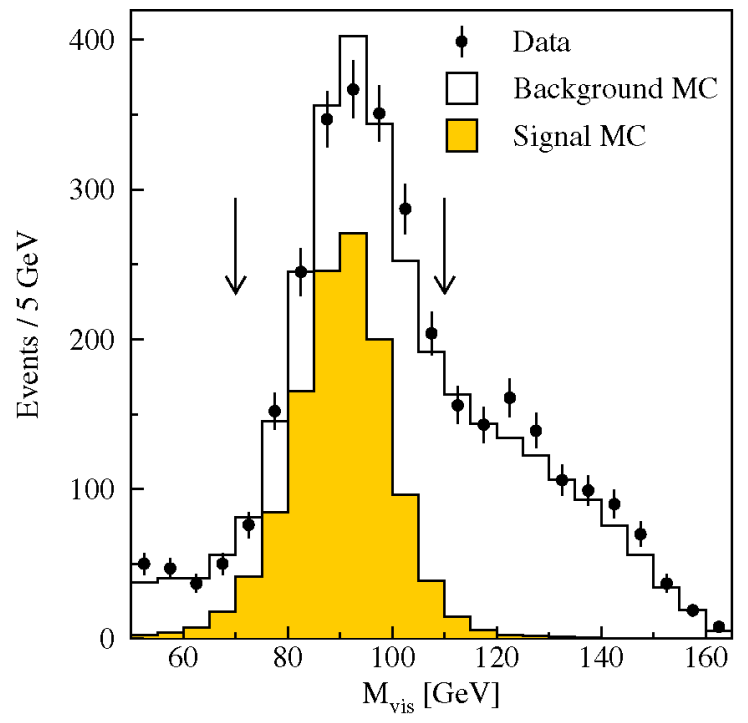

Fig. 3. Distribution of the visible mass for data and Monte Carlo after the application of all other selection cuts.

and $70 \mathrm{GeV}<M_{\text {vis }}<110 \mathrm{GeV}$, as shown in Figs. 2(d) and 3 , respectively. The former criterion is extremely efficient in removing the background from one-photon radiative return to the $\mathrm{Z}$ boson, which is characterised by a larger boost than the signal and therefore a smaller jet opening-angle.

After these selection criteria, 1672 events are selected in data while 1684 are expected from Monte Carlo simulations, of which $61 \%$ are from signal, and $39 \%$ from background, as detailed in Table 2. Three quarters of the background are due to $\mathrm{e}^{+} \mathrm{e}^{-} \rightarrow \mathrm{q} \overline{\mathrm{q}}$ events which do not satisfy the signal definition (1)(5). The remaining background is due to four-fermion
Table 3

Systematic uncertainties on the signal cross section

\begin{tabular}{ll}
\hline Source & Effect (\%) \\
\hline Selection criteria & 2.3 \\
Jet energy scale & 3.0 \\
Jet angle & 3.0 \\
ISR/FSR interference & 1.2 \\
Background normalisation & $1.4-4.4$ \\
Monte Carlo statistics & $1.2-3.9$ \\
Total & $5.3-7.7$ \\
\hline
\end{tabular}

production and hadron production in two-photon collisions. The average signal efficiency is $31 \%$.

The distribution of $M_{\mathrm{vis}}$, shown in Fig. 3, presents a clear enhancement at $m_{Z}$, as expected for signal events.

\section{Systematic uncertainties}

Several sources of possible systematic uncertainties are considered, and their effects are summarised in Table 3 .

Monte Carlo simulations might not perfectly reproduce the tails of the variables used in the event selection owing to, for instance, non-linearity in the modelling of the calorimeter response or a bias in the determination of jet directions close to the edge of fiducial volumes. To assess this effect, the analysis is repeated by removing one selection criterion at a time. In addition, a $0.5 \%$ uncertainty in the jet energy-scale and a $2 \%$ uncertainty in the determination of the jet angles are also considered. 
The signal and background events from the $\mathrm{e}^{+} \mathrm{e}^{-} \rightarrow \mathrm{q} \overline{\mathrm{q}}$ process are generated taking into account the interference between ISR photons and those emitted in the final state. The analysis is repeated by using a Monte Carlo sample without this interference and the difference with the original result is used as an extreme systematic uncertainty on the modelling of this phenomenon.

The cross sections are measured by assuming a fixed background level, as discussed below. Uncertainties in the background cross sections are therefore a possible source of systematic uncertainty, which is estimated by repeating the analysis with a variation of $10 \%$ for the cross section of the $\mathrm{e}^{+} \mathrm{e}^{-} \rightarrow \mathrm{e} v_{\mathrm{e}} \mathrm{q}^{\prime}$ process, $5 \%$ for $\mathrm{e}^{+} \mathrm{e}^{-} \rightarrow \mathrm{q} \overline{\mathrm{q}}$ events classified as background, $5 \%$ for the $\mathrm{e}^{+} \mathrm{e}^{-} \rightarrow \mathrm{ZZ}$ process, $5 \%$ for the $\mathrm{e}^{+} \mathrm{e}^{-} \rightarrow \mathrm{Ze}^{+} \mathrm{e}^{-}$process and $0.5 \%$ for $\mathrm{W}$-boson pair production.

Finally, statistical uncertainties related to the limited amount of Monte Carlo events used to describe the signal and the background processes are included as systematic uncertainties. The total systematic uncertainty on the signal cross section varies between 5.3\% and $7.7 \%$, depending on the centre-of-mass energy.

\section{Results}

The signal cross sections are determined for each centre-of-mass energy by fitting the observed distributions of $M_{\mathrm{vis}}$. Two components are considered, both with a shape fixed to Monte Carlo expectations: a signal component with a free normalisation, and a background component with fixed normalisation. The results are listed in Table 2 and plotted in Fig. 4, together with the corresponding statistical and systematic uncertainties. A good agreement with the predictions of the KK2f Monte Carlo, also given in Table 2 and Fig. 4 , is observed. These predictions have an uncertainty of $3 \%$, which includes a statistical component and the uncertainty from higher-order corrections, estimated following the suggestions in Ref. [10].

To further compare the results and the expectations, the ratio between the measured, $\sigma$, and the expected, $\sigma_{\text {th }}$, values of the cross section is calculated for each centre-of-mass energy. These values are then averaged, by assuming all systematic uncertainties to be fully correlated, with the exception of those due to the

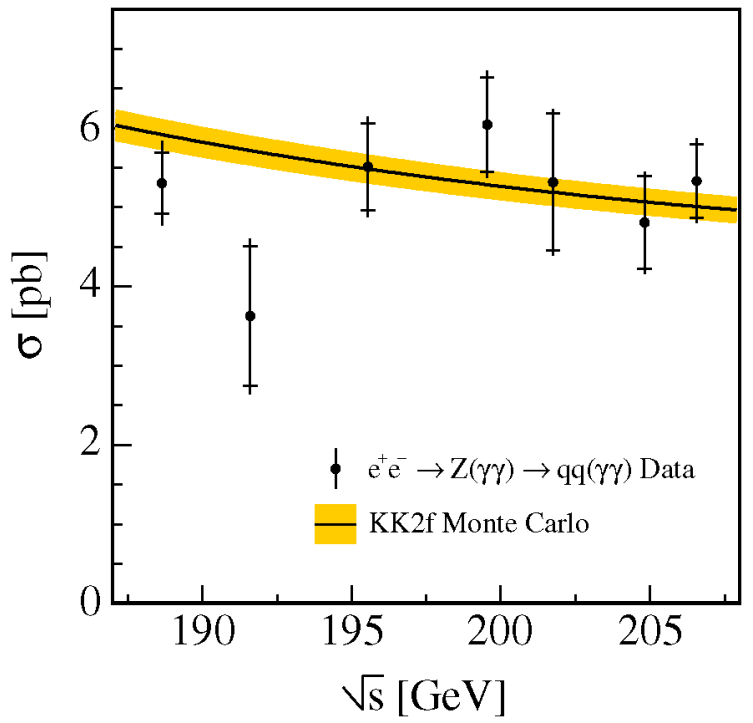

Fig. 4. Measured cross sections for the various centre-of-mass energies, indicated by the points, compared with the Standard Model predictions, indicated by the band. The bars on the point show the sum in quadrature of the statistical and systematic uncertainties. The inner bars represent the statistical uncertainties. The width of the band corresponds to an uncertainty of $3 \%$ on the predictions, derived as discussed in the text.

limited Monte Carlo statistics. The result is

$\sigma / \sigma_{\text {th }}=0.98 \pm 0.04 \pm 0.06$

where the first uncertainty is statistical and the second systematic.

In conclusion, the cross section of the process $\mathrm{e}^{+} \mathrm{e}^{-} \rightarrow \mathrm{Z} \gamma \rightarrow \mathrm{q} \overline{\mathrm{q}} \gamma \gamma$, where the two photons are emitted in the phase space defined by the criteria (1)(5), is measured with an accuracy of 7\% and is well reproduced by the current simulations of ISR in hadronic events. This finding validates the estimate of the background from events with two back-to-back jets with mass close to the mass of the $\mathrm{Z}$ boson both in the searches for Higgs bosons of the Standard Model and beyond and for other manifestations of new physics.

\section{References}

[1] L3 Collaboration, M. Acciarri, et al., Phys. Lett. B 479 (2000) 101 ;

L3 Collaboration, P. Achard, et al., Measurement of hadron and lepton pair production at $192<\sqrt{s}<209 \mathrm{GeV}$ at LEP, in preparation. 
[2] ALEPH Collaboration, R. Barate, et al., Eur. Phys. J. C 12 (2000) 183;

DELPHI Collaboration, P. Abreu, et al., Phys. Lett. B 485 (2000) 45;

OPAL Collaboration, G. Abbiendi, et al., Eur. Phys. J. C 33 (2004) 173.

[3] S. Eidelman, et al., Phys. Lett. B 592 (2004) 1.

[4] L3 Collaboration, P. Achard, et al., Phys. Lett. B 597 (2004) 119.

[5] L3 Collaboration, P. Achard, et al., Phys. Lett. B 585 (2004) 42 ;

OPAL Collaboration, G. Abbiendi, et al., Phys. Lett. B 604 (2004) 31.

[6] L3 Collaboration, M. Acciarri, et al., Phys. Lett. B 478 (2000) 39.

[7] L3 Collaboration, M. Acciarri, et al., Phys. Lett. B 505 (2001) 47 ;

L3 Collaboration, P. Achard, et al., Phys. Lett. B 540 (2002) 43 ;

OPAL Collaboration, G. Abbiendi, et al., Phys. Rev. D 70 (2004) 032005.

[8] L3 Collaboration, B. Adeva, et al., Nucl. Instrum. Methods A 289 (1990) 35;

L3 Collaboration, O. Adriani, et al., Phys. Rep. 236 (1993) 1; J.A. Bakken, et al., Nucl. Instrum. Methods A 275 (1989) 81;

O. Adriani, et al., Nucl. Instrum. Methods A 302 (1991) 53;

B. Adeva, et al., Nucl. Instrum. Methods A 323 (1992) 109;

K. Deiters, et al., Nucl. Instrum. Methods A 323 (1992) 162;

M. Chemarin, et al., Nucl. Instrum. Methods A 349 (1994) 345;
M. Acciarri, et al., Nucl. Instrum. Methods A 351 (1994) 300; G. Basti, et al., Nucl. Instrum. Methods A 374 (1996) 293;

A. Adam, et al., Nucl. Instrum. Methods A 383 (1996) 342.

[9] JETSET version 7.4 and PYTHIA versions 5.722 and 6.1 are used;

T. Sjöstrand, preprint CERN-TH/7112/93 (1993), revised 1995;

T. Sjöstrand, Comput. Phys. Commun. 82 (1994) 74;

T. Sjöstrand, Comput. Phys. Commun. 135 (2001) 238;

T. Sjöstrand, hep-ph/0108264.

[10] KK2f version 4.13 is used;

S. Jadach, B.F.L. Ward, Z. Wạs, Comput. Phys. Commun. 130 (2000) 260.

[11] PHOJET version 1.05 is used;

R. Engel, Z. Phys. C 66 (1995) 203;

R. Engel, J. Ranft, Phys. Rev. D 54 (1996) 4244.

[12] KORALW version 1.33 is used;

M. Skrzypek, et al., Comput. Phys. Commun. 94 (1996) 216; M. Skrzypek, et al., Phys. Lett. B 372 (1996) 289.

[13] EXCALIBUR version 1.11 is used; F.A. Berends, R. Kleiss, R. Pittau, Comput. Phys. Commun. 85 (1995) 437.

[14] GEANT version 3.15 is used;

R. Brun, et al., preprint CERN DD/EE/84-1 (1985), revised 1987.

[15] H. Fesefeldt, RWTH Aachen Report PITHA 85/02 (1985).

[16] S. Catani, et al., Phys. Lett. B 269 (1991) 432;

S. Bethke, et al., Nucl. Phys. B 370 (1992) 310. 